\title{
Performance improvement initiative: prevention of surgical site infection (SSI)
}

Wai Khuan Ng, Nawal Awad

Mafraq Hospital

\begin{abstract}
Mafraq Hospital performs an average of 10,000 surgeries every year. The impact of having high volume high risk surgical procedures calls for the need to ensure safe surgery and a prevention of surgical site infection (SSI). SSI represents a significant portion of healthcare-associated infections (HAIs). The impact on morbidity, mortality, and cost of care has resulted in identifying the need to reduce SSI as a top priority to prevent healthcare associated infections. The good news is that the majority of SSIs are preventable.

Mafraq Hospital performs a range of surgical procedures that covers 14 surgical specialties. The infection prevention and control team performs surveillance for SSI for all patients who undergo operative procedure included in Centers for Disease Control and Prevention (CDC) National Healthcare Safety Network (NHSN) Operative Procedure Category (40 surgical procedures). Out of the 40 CDC NHSN listed, 33 operative procedures were performed at Mafraq Hospital, of which 17 were reported with SSI for 2013 and 2014. Surgical site infection has implicated an increase average length of stay from seven to 10 additional postoperative hospital days and additional costs of AED 10,000 to AED 100,000/SSI depending on procedure and pathogen.

A multidisciplinary team was formed to develop and implement measures to reduce/eliminate surgical site infection, as well as evaluate and monitor compliance. Hence a group of multidisciplinary teams were initiated to analyse the results, find out the gaps, and implement a quality improvement project to correct the deficits. Recommendations for appropriate improvement measures were formed on evidence-based international guidelines from the Institute for Healthcare Improvement $(\mathrm{IHI})$ and $\mathrm{CDC}$. Evidence based practice supports that many of the causes of surgical site infection can be prevented with proper medical attention and care.
\end{abstract}

\section{Problem}

Currently Mafraq Hospital performs a range of surgical procedures that covers 14 surgical specialties. The infection prevention and control (IPC) team performs surveillance for SSI for all patients who undergo operative procedure included in Centers for Disease Control and Prevention (CDC) National Healthcare Safety Network (NHSN) Operative Procedure Category (40 surgical procedures).[1] For each of these surgical patients the following is monitored: Patient's medical record number, procedure date, American Society of Anaesthesiologists (ASA) score, wound class, duration of surgery (hour and minutes).[1]

Out of the 40 CDC NHSN listed operative procedures 33 were performed at Mafraq Hospital, of which 17 operative procedures were reported with SSI for 2013 and 2014. The SSI rate is calculated by dividing the total number of SSI per procedures by the total number of procedures performed as per CDC NHSN $x$ by 100 (constant).[1]

\section{Background}

Mafraq Hospital performs an average of 10,000 surgeries every year. The impact of having high volume high risk surgical procedures calls for the need to ensure safe surgery and prevent surgical site infection (SSI) [2].
Surgical site infection (SSI) represents a significant portion of healthcare-associated infections (HAls) [1, 2]. The impact on morbidity, mortality, and cost of care has resulted in SSI reduction being identified as a top priority to prevent HAls [1, 2]. The majority of SSIs are preventable [1,2]. Worldwide attention to safer surgery including prevention of SSI led to the development of the World Health Organization (WHO) Surgical Safety Checklist demonstrating the importance of teamwork and communication in addition to evidence-based care for preventing SSI [3]

\section{Baseline measurement}

There were 72 operative procedures identified with SSI in 2013 , while 21 reported in 2014 (table 1). As shown in graph 1, these SSI cases for these two years were classified by categories. Table 2 showed the SSI rate for each procedure compared the SSI benchmark by CDC NHSN for those procedures. Overall, the SSI mean rate for 2013 was reported to be $2.0 \%$ as indicated in graph 2 and an increase of SSI rate was reported from 1.7\% in Q1 2013 to $2.8 \%$ in Q3 2013 represented in graph 3.

See supplementary file: ds4912.pdf - "Baseline Measurement SSI Rate 2013 - 2014"

\section{Design}




\section{BMJ Quality Improvement Reports}

A multidisciplinary team consisting of the following: infection prevention and control (IPC), nursing, operating room (OR) nurse manager, operating room nurse educator, pharmacy, and physicians (infectious diseases, anesthesia, and surgery) was formed to develop and implement measures to reduce or eliminate surgical site infection, evaluate and monitor compliance.

Recommendations for appropriate improvement measures were based on evidence-based international guidelines (IHI and CDC).[4, 5]

Surgical site infection has significantly implicated an increase average length of stay from seven to 10 additional postoperative hospital days and additional costs of AED 10,000 to AED $100,000 /$ SSI depending on procedure and pathogen.[1]

Hence a group effort from multidisciplinary team was initiated to analyze the results, find out the gaps and implement a quality improvement project to correct the deficits. Evidence based practice supports that many of the causes of surgical site infection can be prevented with proper medical attention and care.[1-4]

\section{Strategy}

PDCA cycle 1: To clarify current knowledge, the multidisciplinary team members met and researched the possible causes of surgical site infection. The 72 cases with SSI were analyzed and 14 factors were identified as noncompliance with prevention of SSI. In light of this, the team planned the following:

1. Communicate the project aim and objectives to all key stakeholders of surgical service

2. Revise the clinical practice guidelines about surgical antimicrobial prophylaxis based on scientific evidence

3. Educate all surgical specialties about the recommended clinical practice guidelines

4. Implement the hospital-approved antimicrobial prophylaxis guidelines and monitor compliance

5. Review the intraoperative practice about surgical scrub and site preparation

6. Develop SSI bundle, and educate all surgical service members on the bundle

7. Implement the bundle, evaluate and monitor compliance

8. Develop preoperative education material for surgical patients

9. Report and feedback monthly SSI rate to surgical specialties

10. Carry out root cause analysis (RCA) for reported SSI.

The following prevention strategies were implemented:

1. Preoperative screening to ensure patients is medically fit for surgery

2. Reinforcement of the preoperative patient education regarding hair removal using clippers (if hair removal is required) and the use of an antiseptic agent for bathing (ie, chlorhexidine gluconate) prior surgery

3. Observation of the skin preparation technique followed pre surgery in the operating room (OR)
4. Administration of antimicrobial prophylaxis in accordance with evidence based standards and guidelines

5. Limit OR traffic when operative procedure is ongoing

6. Maintenance of normothermia of all post-operative patients in the pre holding area, intra operatively and during the recovery phase

7. Improve communication of SSI rates with key stakeholders

8. Performing surgical wound dressing under strict aseptic technique.

Noncompliance with the above identified factors was observed among the surgical team. Hence, OR leadership took ownership to reinforce the implementation of the SSI Bundle. The assistant director of surgical services, OR manager, and OR nurse educator reinforced the SSI bundle and the recommended clinical practice guidelines during the surgical department meeting, and the strategies were then trialled again as PDCA cycle 2.

PDCA cycles 2 to 4: Over a six week period, the initiated interventions were monitored, prompt feedback on SSI rates was provided to relevant surgical specialties, and recommendations to comply with clinical practice guidelines and SSI bundle were consistently reinforced. Gradually, the strategies were implemented to all patients undergoing operative procedures.

PDCA cycle 5: An educational session for all surgeons was organized to raise awareness of the prevention of SSI using evidence-based strategies. Electronic SSI bundle compliance tool was developed.

PDCA cycle 6: SSI rates feedback and factors of non-compliance were made as standing item during the department meeting and in the agenda for OR Committee. All new surgical healthcare workers (physicians, anaesthetists and nurses) were oriented about SSI bundle during induction. All reported SSI was discussed in the mortality and morbidity committee. Throughout the whole process, data were continually collected and collated by the infection prevention and control team.

\section{Results}

Results of SSI showed reduction in SSI rate from $2.8 \%$ in the third quarter 2013 to $1.0 \%$ in the second quarter 2014 (graph 4). As indicated in graph 5,6 , and $7,100 \%$ of surgical antimicrobial prophylaxis (AMP) was selected, administered, and discontinued as per hospital-approved clinical guideline. Besides this, the aim and objectives of the performance improvement project was communicated to all surgical departments during their department morning meeting

The surgical service departments were also visited by the infection prevention and control team where the findings of the root cause analysis were shared with all surgeons, anaesthetists, and surgical nurses. The root cause analysis (RCA) tool was developed and communicated to all surgical departments. RCA was carried out on every reported SSI with the involvement of the respective chief of specialty. 


\section{BMJ Quality Improvement Reports}

See supplementary file: ds4934.pdf - "Post Measurement SSI Rate 2013 - 2014"

\section{Lessons and limitations}

A number of lessons have been learnt from carrying out this project:

1. If we are to substantially minimize errors and improve quality, we must create systems and care processes that anticipate inevitable human errors and either prevent or counteract them before they generate harm

2. Changes happen only with the involvement of key stakeholders. When implementing an intervention, the multidisciplinary team that the intervention could impact upon should be considered since success will require a multifaceted strategy

3. As there have been an increased focus and numerous interventions, it can be challenging to justify which factor has actually contributed to the decrease of SSI rate

4. Process communication and result feedback are vital to enhance performance improvement. Although we consistently emphasized the importance of getting buy-in from key stakeholders in making change, the involvement of certain key stakeholders (eg, surgeons) was limited due to patient commitment.

\section{Conclusion}

A recent prevalence study found that surgical site infections were the most common healthcare-associated infection (HAls), accounting for $31 \%$ of all HAls among hospitalized patients.[6] SSI is associated with a mortality rate of $3 \%$, and $75 \%$ of SSIassociated deaths are directly attributable to the SSI.[7] Surveillance of SSI with feedback of appropriate data and possible contributing factors to the key stakeholders including surgeons has been shown to be an important component of strategies to reduce SSI risk [5, 8-10]. Multidisciplinary effort is also crucial to ensure system and care processes are in place to prevent and compensate inevitable human errors.

\section{References}

1. Centers for Disease Control and Prevention (CDC) National Healthcare Safety Network (NHSN)(2015), Surgical site infection: 1-27

(http://www.cdc.gov/nhsn/PDFs/pscManual/9pscSSIcurrent. pdf).

2. Association for Professionals of Infection Control and Epidemiology (APIC) Guide (2010), Elimination of orthopaedic surgical site infections: 1-80

(http://www.apic.org/Resource/EliminationGuideForm/34e0 3612-d1e6-4214-a76b-e532c6fc3898/File/APIC-Ortho-

Guide.pdf).

3. World Health Organization (WHO) Guideline (2009), Safe surgery saves lives: 1-133 (http://whqlibdoc.who.int/publications/2009/9789241598552 eng.pdf?ua=1).
4. Institute for Healthcare Improvement (IHI) (2012), Prevention of surgical site infection: 1-28 (http://www.ihi.org/resources/Pages/Tools/HowtoGuidePrev entSurgicalSitelnfection.aspx).

5. Mangram AJ, Horan TC, Pearson ML, Silver LC, Jarvis WR. Guideline for prevention of surgical site infection. Infect Control Hosp Epidemiol 1999;20(4):247-78.

6. Magill SS, Hellinger W, Cohen J, Kay R, Bailey C, Boland B, Carey D, de Guzman J, Dominguez K, Edwards J, Goraczewski L, Horan T, Miller M, Phelps M, Saltford R, Seibert J, Smith B, Starling P, Viergutz B, Walsh K, Rathore M, Guzman N, Fridkin S. Prevalence of healthcareassociated infections in acute care hospitals in Jacksonville, Florida. Infect Control Hosp Epidemiol 2012;33(3):283-91.

7. Awad SS. Adherence to surgical care improvement project measures and post-operative surgical site infections. Surg Infect (Larchmt) 2012;13(4):234-7.

8. Condon RE, Schulte WJ, Malangoni MA, AndersonTeschendorf MJ. Effectiveness of a surgical wound surveillance program. Arch Surg 1983;118(3):303-7.

9. Consensus paper on the surveillance of surgical wound infections, The Society for Hospital Epidemiology of America, The Association for Practitioners in Infection Control, The Centers for Disease Control, The Surgical Infection Society. Infect Control Hosp Epidemiol 1992;13(10):599-605.

10. Haley RW, Culver DH, White JW, Morgan WM, Emori TG, Munn VP, Hooton TM. The efficacy of infection surveillance and control programs in preventing nosocomial infections in US hospitals. Am J Infect Control 1985;121(2):182-205.

\section{Declaration of interests}

Nothing to declare.

\section{Acknowledgements}

We would like to thank

- Ms Leonie Van Zyl and Ms Martha Jacoba Weideman in educating all surgical service members on SSI bundle

- Mafraq infection prevention and control team in collecting the data

- Ms Filestin Jaber (quality officer) and Ms Nazik Abdelaziz El Hassan (quality officer) in guiding the PDCA cycles.

\section{Ethical approval}

Ethical approval is not required for this project as the work was deemed an improvement study and not a study on human subjects. 